Rolinson, G. N. (1952). J. gen. Microbiol. 6, 336-343.

\title{
Respiration of Penicillium chrysogenum in Penicillin Fermentations
}

\author{
By G. N. ROLINSON \\ Research Department, Bacteriology Division, Boots Pure Drug Co. Ltd., Nottingham
}

SUMMARY: Spore-inoculated penicillin fermentations using Penicillium chrysogenum W 48-701 were carried out in two different types of equipment: stirred and aerated laboratory fermentors, or shaken flasks. The respiration of the mycelium was measured in Warburg manometers. The maximum oxygen demand in the fermentations occurred at approximately $40 \mathrm{hr}$. and the maximum $Q_{0_{2}}$ of the mycelium at $24 \mathrm{hr}$. or earlier. During the whole phase of penicillin formation, respiration decreased steadily. Mycelium grown in the stirred and aerated vessels showed the highest respiration rate. In the shaken flasks the rate of solution of oxygen in the medium was found by polarographic methods to be insufficient to allow a respiration rate as high as that which occurred in the stirred and aerated vessels. Respiration in shaken flasks, however, was not directly limited by the rate of solution of oxygen but by the amount and kind of the respiratory enzymes which appear to be synthesized in proportion to the concentration of dissolved oxygen present during early growth.

Whereas the respiratory quotient (R.Q.) of mycelium from shaken-flask fermentations remained approximately unity throughout the fermentation, in the stirred and aerated vessels the R.Q. of the mycelium fell from unity to as low as 0.57 , during the fermentation. The degree of aeration therefore appears to influence not only the quantity but also the kind of respiratory enzymes synthesized.

Studies on the oxygen requirements of Penicillium chrysogenum in submerged fermentations have been largely concerned with determining the optimum conditions of aeration for maximum penicillin production (Brown \& Peterson, 1950; Bartholomew, Karow, Sfat \& Wilhelm, 1950). Less attention has been paid to the respiratory activities of the mycelium, particularly with respect to different types of fermentation equipment. The experiments reported here were designed to determine the oxygen demands of mycelium throughout fermentations and the effect of different degrees of aeration on the respiration of the organism.

\section{METHODS}

Fermentation methods. Laboratory fermentors of the type described by Lumb \& Fawcett (1951) containing 31 . of medium were operated with an air flow of $2 \mathrm{vol} . / \mathrm{vol}$. medium/min. and a stirring speed of $1000 \mathrm{r} . \mathrm{p} . \mathrm{m}$. Shakenflask fermentations were carried out with $75 \mathrm{ml}$. medium in $250 \mathrm{ml}$. conical Pyrex flasks on a rotary shaker having a speed of 220 r.p.m. and a throw of $1 \frac{1}{2}$ in. All fermentations were carried out at $24-26^{\circ}$.

The medium used in all the experiments contained : corn steep liquor, solids basis, $1.5 \%$; lactose, $3.0 \%$; glucose, $0.5 \%$; $\mathrm{NaNO}_{3}, 0.3 \%$; $\mathrm{KH}_{2} \mathrm{PO}_{4}, 0.05 \%$; $\mathrm{Na}_{2} \mathrm{SO}_{4}, 0.1 \% ; \mathrm{pH}$ value adjusted to $5 \cdot 8$. Phenylacetic acid was added to the stirred and aerated fermentations in additions of $0.05 \%$ every $12 \mathrm{hr}$. starting at the 24th hr. In the shaken-flask fermentations a concentration of $0.25 \%$ 
phenylacetic acid was usually included in the medium before autoclaving. While penicillin production was then very satisfactory, early growth in the shaken flasks appeared to be retarded by the initial presence of phenylacetic acid. Since the purpose of the present work was to compare the respiratory activities of mycelium rather than to produce maximum penicillin titres, phenylacetic acid was consequently omitted from all the shaken-flask fermentations described here. Lard oil containing $2.5 \%$ cetyl alcohol was used as an antifoam agent when required. All fermentations were inoculated with a spore suspension of Penicillium chrysogenum W 48-701.

Manometric methods. Conventional Warburg techniques were used as described by Umbreit, Burris \& Stauffer (1945).

Determination of the efficiency of aeration in the fermentation equipment. The polarographic methods described by Wise (1951) were used. Aeration is measured in terms of a constant, $\phi_{L}$, which is dependent on the rate of air flow, agitation, shape of vessel, etc. The value of $\phi_{L}$ for different conditions of aeration was determined by the gassing-out method of Wise (1951). The value of $\phi_{L}$ characterizes the efficiency of aeration in a particular vessel for a given air flow and rate of agitation. The minimum value of $\phi_{L}$ necessary to ensure an adequate rate of solution of oxygen depends on the oxygen demand of the organism. It was shown by Wise (1951) that if the rate of solution of oxygen in a liquid is expressed by the equation

$$
\frac{d C}{d t}=\phi_{L}\left(C_{e}-C\right)
$$

where $C$ is the concentration of dissolved oxygen at time $t$ and $C_{e}$ the concentration of oxygen when the solution is saturated with air, the maximum rate at which oxygen can dissolve is given by $\phi_{L} C_{e}$, since the rate of solution of oxygen will be maximum when $C=0$. Therefore if $k_{0}$ represents the oxygen demand of mycelium the fermentation will be adequately aerated if $\phi_{L} C_{e}>k_{0}$, hence the minimum value of $\phi_{L}$ necessary for adequate aeration can be obtained. The value of $k_{0}$ expressed in arbitrary polarograph units is obtained by plotting the uptake of dissolved oxygen in the polarograph cell against time. $C_{e}$ can be obtained by filtering off the mycelium and saturating the sample with air. By these methods the actual value of $\phi_{L}$ in a particular fermentation can be obtained and also the minimum value of $\phi_{L}$ necessary in that fermentation to ensure an adequate supply of oxygen to the mycelium. It is thus possible to assess the state of aeration in a fermentation.

Mycelial weight. Dry mycelial weights were determined by filtering a measured volume of a fermentation sample on a tared filter-paper in a Buchner funnel, drying overnight at $80^{\circ}$ and weighing.

\section{RESULTS}

\section{Aeration conditions in the fermentation equipment}

The values of $\phi_{L}$ (defined above) for the stirred and aerated vessels and shaken flasks are shown in Table 1 . In all the fermentations in the stirred and aerated vessels stirring was at 1000 r.p.m. and air flow 2 vol./vol. medium $/ \mathrm{min}$. 
It will be seen from Table 1 that the value of $\phi_{L}$ under these conditions was $>10$. At the time of peak oxygen demand (determined from preliminary manometric experiments) the minimum value of $\phi_{L}$ necessary to ensure an adequate rate of solution of oxygen to meet the demand of the mycelium was found to be $\mathbf{0 . 7 8}$ (average of three fermentations). The rate of solution of oxygen in such fermentations therefore was greatly in excess of the maximum demand. Even at the peak oxygen demand the fermentation medium was 80-90\% saturated with air.

It will be seen from Table 1, however, that in shaken-flask fermentations the value of $\phi_{L}$ was $\mathbf{0 . 4 3}$. The rate of solution of oxygen was therefore not sufficient to meet a demand as high as that shown by mycelium from the stirred and aerated fermentations, for which a minimum value of 0.78 was required. The oxygen uptake of mycelium in shaken flasks therefore must be lower than that of mycelium in the highly aerated vessels. From these experiments it is clear that the two types of fermentation equipment gave widely different conditions of aeration.

Table 1. Values of $\phi_{L}$ in stirred and aerated vessels and in shaken flasks

\begin{tabular}{ccc}
\multicolumn{3}{c}{ Stirred and aerated vessels } \\
$\begin{array}{c}\text { Stirring } \\
\text { (r.p.m.) }\end{array}$ & Air flow & (vol./vol./min.) \\
1000 & 2 & $\phi_{i}$ \\
740 & 2 & 10 \\
730 & 1 & 7 \\
420 & 1 & $3 \cdot 2$ \\
350 & 1 & $1 \cdot 12$ \\
300 & 1 & $0 \cdot 46$ \\
850 & $0 \cdot 2$ & $0 \cdot 3$ \\
550 & 0.2 & $1 \cdot 4$ \\
440 & 0.2 & $0 \cdot 65$ \\
& Shaken flasks & $0 \cdot 48$ \\
(r.p.m.) & Throw & $\phi_{L}$ \\
220 & 1.5 in. & $0 \cdot 43$
\end{tabular}

\section{Oxygen uptake in the stirred and aerated vessels}

Samples were removed at intervals from the fermentation vessels and the uptake of oxygen measured in Warburg manometers at the temperature of the fermentation at that time. To ensure that the rate of solution of oxygen in the Warburg flasks was not a limiting factor, the rocking rate of the flasks was increased until no further increase in the rate of oxygen uptake occurred. With samples of $1 \mathrm{ml}$. fermentation liquor and $1 \mathrm{ml}$. sterile distilled water/ flask it was found that a rocking rate of 120 strokes $/ \mathrm{min}$. provided an ample margin in the rate of solution of oxygen for all samples. A measured volume of the sample was used to determine mycelial dry weight.

The results of a typical experiment are shown in Fig. $1 A$. The oxygen demand of the fermentation rose sharply during the early stages and reached a maximum at 36-48 hr. After this, despite continued mycelial growth the 
oxygen demand decreased steadily and was at less than half the maximum rate when the accumulation of penicillin was at a maximum, at about 4 days. Penicillin production did not begin until $c .36 \mathrm{hr}$. and then occurred at a uniform rate until $c$. the $96 \mathrm{th} \mathrm{hr}$. Over almost the entire period of penicillin production the oxygen demand fell steadily, and during the later stages of penicillin formation the weight of mycelium also decreased. In these fermentations the production of penicillin was clearly not a direct function of mycelial growth or of oxygen uptake but was rather the result of independent reactions which may, however, be influenced by the earlier respiratory activities of the mycelium.

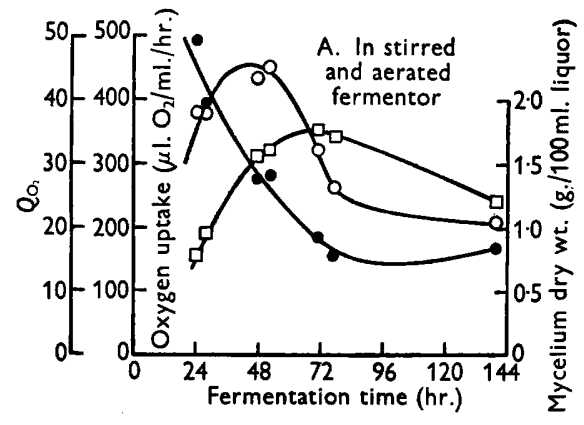

$\boldsymbol{A}$

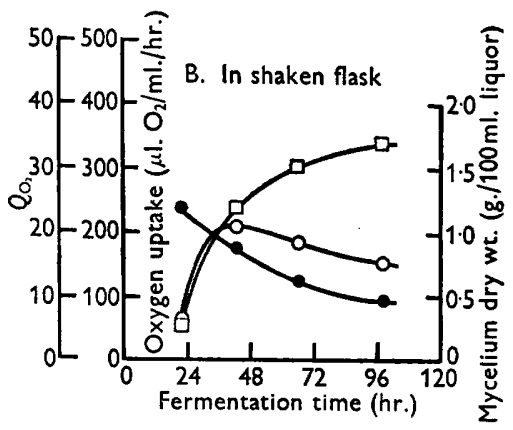

$\boldsymbol{B}$

Fig. 1. Oxygen uptake and growth of Penicillium chrysogenum W 48-701 in spore-inoculated penicillin fermentations. Oxygen uptake $/ \mathrm{ml}$. brew $=\mathrm{O}-\mathrm{O}-\mathrm{O} ; Q_{\mathrm{O}_{2}}=\mathrm{O}-\mathrm{O}-\mathrm{O}$ growth $=\square-\square-\square . A$, in laboratory fermentor stirred at 1000 r.p.m. with air flow, 2 vol./vol. liquor/min.; $B$, in shaken flask.

The respiration rate of mycelium as distinct from the oxygen uptake/ml. fermentation liquor is expressed as $\mu \mathrm{l} . \mathrm{O}_{2}$ taken $\mathrm{up} / \mathrm{mg}$. dry mycelium $/ \mathrm{hr}$. $\left(Q_{\mathrm{O}_{3}}\right)$ in Fig. 1. It will be seen that the $Q_{\mathrm{O}_{2}}$ was highest at the earliest time of sampling, i.e. $24 \mathrm{hr}$. and continued to decrease steadily throughout the rest of the fermentation period. At the time of maximum penicillin production the rate of respiration was relatively low.

\section{Oxygen uptake in shaken flasks}

Shaken-flask fermentations were sampled at intervals and the oxygen uptake measured as in the experiments with the highly aerated fermentations. The results of a typical experiment are shown in Fig. $1 B$. Although the peak oxygen demand again occurred at $36-48 \mathrm{hr}$. the rate of oxygen uptake was very much lower than in the highly aerated vessels. It was evident that this would be so from the rate of solution of oxygen in the shaken flasks. In the latter the maximum rate of oxygen uptake was only half that of mycelium from the highly aerated fermentations. In addition, the rate of growth was slower and so was the rate of penicillin production. 


\section{The effect of aeration on the respiratory quotient}

In the highly aerated fermentations the volume of carbon dioxide evolved during the first $36 \mathrm{hr}$. corresponded closely to the volume of oxygen taken up thus giving a respiratory quotient (R.Q.) of approximately unity. After $36 \mathrm{hr}$., however, the R.Q. fell [and reached a value of $0.7-0.6$ during the period of maximum rate of penicillin production. On occasion the R.Q. was as low as $\mathbf{0 . 5 7}$ after only $\mathbf{5 0} \mathrm{hr}$. In contrast with this effect, the evolution of carbon dioxide in the shaken-flask fermentations closely paralleled the oxygen uptake throughout the greater part of the fermentation. An R.Q. of approximately 1.0 was therefore maintained without the pronounced decrease which occurred in the highly aerated fermentations. The R.Q. values for two typical experiments are shown in Table 2.

Table 2. The respiratory quotients of penicillin fermentations in shaken flasks and stirred and aerated vessels

\begin{tabular}{ccccc}
\multicolumn{2}{c}{ Shaken flasks } & \multicolumn{3}{c}{ Stirred and aerated vessels } \\
Hr. & R.Q. & & Hr. & R.Q. \\
21 & 1.27 & & & \\
26 & 1.12 & & 24 & $1 \cdot 0$ \\
43 & 1.05 & & 46 & 0.87 \\
50 & 1.02 & & 51 & 0.80 \\
66 & 0.94 & & 69 & 0.72 \\
74 & 1.05 & & & \\
98 & 0.81 & & 94 & 0.60
\end{tabular}

The continued high R.Q. value in shaken flasks and the falling values in the highly aerated fermentations were shown in all the experiments carried out and appeared to represent some fundamental difference in the respiratory activities in the two types of fermentation.

\section{The effect of aeration on the synthesis of respiratory enzymes}

It will be seen from Fig. $1 A \& B$ that the rate of respiration $\left(Q_{\mathrm{O}_{2}}\right)$ of mycelium grown in shaken flasks was very much lower than that of mycelium from the highly aerated fermentations. The average of the maximum $Q_{O_{2}}$ values of three such shaken-flask fermentations was $37 \cdot 0$, whereas that of three parallel fermentations in the stirred and aerated vessels was 71.7. All the measurements of the respiration rates, however, were carried out under conditions which ensured an excess of available oxygen. Despite this excess of oxygen in the Warburg manometers the mycelium obtained from fermentations in the two types of equipment showed this marked difference in respiration rate.

It would appear therefore that a less active respiratory mechanism is elaborated in the shaken flasks compared with the highly aerated fermentations, and that it is the activity of this mechanism that limits respiration, not the immediate supply of available oxygen. Since a more active respiratory 
mechanism is synthesized under the conditions of the higher degree of aeration it would appear that the concentration of dissolved oxygen during the early period of growth influences the synthesis of respiratory enzymes. Not only the quantity but also the kind of respiratory enzymes synthesized appears to be influenced by the degree of aeration. Attention has already been drawn to the results given in Table 2 which show that different types of metabolism take place in the two different sets of fermentation equipment. A continued high R.Q. was shown by mycelium from the shaken flasks and a lower and decreasing R.Q. by mycelium from the highly aerated fermentations. This

Table 3. The effect of different conditions of available oxygen on the respiration of mycelium from shaken flasks and from stirred and aerated vessels

\begin{tabular}{|c|c|c|c|c|c|c|c|}
\hline \multirow[b]{2}{*}{$\begin{array}{c}\text { Age of } \\
\text { mycelium } \\
\text { (hr.) }\end{array}$} & \multirow{2}{*}{$\begin{array}{l}\text { Manometer } \\
\text { rocking } \\
\text { rate } \\
\text { (strokes/ } \\
\text { min.) }\end{array}$} & \multicolumn{3}{|c|}{ Mycelium from shaken flasks } & \multicolumn{3}{|c|}{$\begin{array}{l}\text { Mycelium from stirred } \\
\text { and aerated vessels }\end{array}$} \\
\hline & & 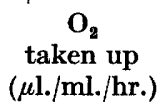 & $\begin{array}{c}\mathrm{CO}_{2} \\
\text { evolved } \\
(\mu \mathrm{l} . / \mathrm{ml} . / \mathrm{hr} .)\end{array}$ & R.Q. & $\begin{array}{c}\mathrm{O}_{\mathbf{2}} \\
\text { taken up } \\
(\mu \mathrm{l} . / \mathrm{ml} . / \mathrm{hr} .)\end{array}$ & $\begin{array}{c}\mathrm{CO}_{2} \\
\text { evolved } \\
(\mu \mathrm{l} . / \mathrm{ml} . / \mathrm{hr} .)\end{array}$ & R.Q. \\
\hline 46 & 150 & 273 & 264 & 0.97 & 426 & 246 & $\mathbf{0 . 5 8}$ \\
\hline 46 & $\mathbf{0}$ & 140 & 144 & $1 \cdot 03$ & 176 & 128 & $0 \cdot 73$ \\
\hline 50 & 150 & 247 & 227 & 0.92 & 421 & 239 & 0.57 \\
\hline 50 & 0 & $98 \cdot 7$ & 95 & 0.96 & 134 & 73 & $0 \cdot 54$ \\
\hline
\end{tabular}

difference in metabolism although apparently resulting in the first place from the difference in aeration is little influenced by the immediate concentration of available oxygen. The respiration of the two types of mycelium under the reverse conditions is shown in Table 3. Mycelium grown under conditions in the high degree of aeration present in the stirred vessels showed the same type of respiration, as indicated by the R.Q., when the oxygen supply was limited in the Warburg flasks by not rocking them as when an excess of dissolved oxygen was provided by rocking the flasks at 150 strokes/min. Conversely the typical R.Q. value of $c$. unity shown by shaken-flask mycelium was also as apparent when an excess of oxygen was provided as when the supply was limited.

The difference in metabolism is therefore not due to the selective functioning of different enzyme systems in response to the immediate supply of oxygen. The degree of aeration in the fermentation appears rather to influence the synthesis of different enzymes so that different enzymic equipments exist in the two types of mycelium.

\section{DISCUSSION}

These experiments show that the oxygen demand does vary considerably as the fermentation proceeds and is greatest at a relatively early stage. The time of peak oxygen demand would probably be even earlier if a vegetative inoculum were used. In shaken-flask fermentations the maximum oxygen demand occurred at approximately the same time as in the highly aerated fermentations, but was, however, considerably lower. In shaken flasks the 
rates of growth and of penicillin production were slower than in the stirred and aerated vessels.

Although a high level of aeration is necessary for maximum penicillin yields, the peak oxygen demand does not coincide with the time of maximum penicillin formation. During penicillin formation, which occurs at an almost uniform rate, the respiration rate decreases steadily. It would appear that high aeration is therefore not directly required for the process of penicillin formation but rather for the formation of the enzyme mechanism necessary for penicillin production and that, once formed, this mechanism does not function in proportion to the subsequent rate of growth or rate of respiration.

The fact that mycelium when grown under conditions of low aeration and subsequently subjected to an increased supply of available oxygen continues to respire at a slower rate than mycelium grown initially under conditions of higher aeration shows that respiration is not directly limited by the immediate supply of available oxygen. Oxygen uptake by $\boldsymbol{P}$. chrysogenum is unaffected by oxygen concentration until very low concentrations are reached. By plotting the rate of uptake of oxygen from solution by mycelium in a sample of liquor in a polarograph cell a concentration below $10 \%$ of the value for saturation with air was reached before the concentration of oxygen began to limit oxygen uptake. If respiration in fermentations with low aeration were limited by the concentration of available oxygen, i.e. the latter being below the critical level, an increase in the concentration of oxygen would be expected to result in an increase in the respiration rate. Since this does not in fact occur, it must be assumed that the immediate concentration of available oxygen is not the limiting factor in respiration. In the fermentations with low aeration the concentration of available oxygen was adequate to permit a higher respiration rate than actually occurred. In such fermentations with low aeration, however, not only was the respiration rate diminished but also the quantity and rate of penicillin production. Thus for optimum penicillin production the rate of solution of oxygen in a vessel must not only ensure that the supply of oxygen is not limiting respiration, since very low concentrations of oxygen ensure this. Respiration is apparently limited by the magnitude of the existing respiratory mechanism. The latter appears to be synthesized in proportion to the concentration of available oxygen during early growth. A high degree of aeration results in a high concentration of dissolved oxygen and it is this concentration that apparently determines the future rate of respiration and possibly the rate of penicillin production also.

The author wishes to express his thanks to Sir Jack Drummond, F.R.S., and Mr C. E. Coulthard for their interest in the work, to Dr M. Lumb and Mr R. Fawcett for providing fermentations on which these investigations were carried out, and to Mr C. K. Mercer and Mr J. Ransom for technical assistance. 


\section{REFERENCES}

Bartholomew, W. H., Karow, E. D., Sfat, M. R. \& Wilhelm, R. H. (1950). Effect of air flow and agitation rates upon fermentation of Penicillium chrysogenum and Streptomyces griseus. Industr. Engng. Chem. 42, 1810.

Brown, W. E. \& Peterson, W. H. (1950). Factors affecting production of penicillin in semi-pilot plant equipment. Industr. Engng. Chem. 42, 1769.

LumB, M. \& FAwCETT, R. (1951). Improvements in experimental fermentors. J. appl. Chem. 1, S94.

Umbreit, W. W., Burris, R. H. \& StaUfFer, J. F. (1945). Manometric Techniques and Related Methods for the Study of Tissue Metabolism. Minneapolis: Burgess Publishing Co.

Wise, W. S. (1951). The measurement of the aeration of culture media. J. gen. Microbiol. 5, 167.

(Received 22 October 1951) 